\title{
A PROPÓSITO DE CRIOLLOS Y MESTIZOS EN EL MÉXICO COLONIAL (SIGLO XVIII)
}

\author{
Alexandre Coello de la Rosa \\ Universitat Pompeu Fabra (UPF-CSIC) \\ (Barcelona, España) \\ ORCID: https:// \\ orcid.org/0000-0001-5079-6180 \\ alex.coello@upf.edu
}

En los últimos años diversos estudiosos —antropólogos, críticos literarios, historiadoresse ban interesado por los conceptos de «raza» y sangre, así como por las diversas categorias étnico-sociales — «mestizo», «criollo», «pardo», «español», «indio», etc. - emergentes en el mundo colonial hispano (siglo XVI-XVIII). Este artículo analiza un texto seminal de 1994 de Juan Carlos Garavaglia y Juan Carlos Grosso para el contexto novohispano, destacando su enfoque histórico y antropológico riguroso (y en especial un análisis sistemático de los datos censales disponibles), lo que les permitió plantear que los grupos étnicos y sociales no eran categorías contrapuestas sino sistemas de representación mutuamente significativos.

Palabras Clave: Mexico, Garavaglia, Grosso, mestizaje, siglo XVIII.

\section{About Criollos and Mestizos in Colonial MeXico (18Th Century)}

In recent years various scholars - anthropologists, literary critics, historians - have been interested in the concepts of «race» and blood, as well as in the different ethnic and social categories — «mestizo», «criollo», «pardo», «Spanish», «Indian», etc. - that emerged in the Hispanic colonial world (XVI-XVIII century). This article analyzes a 1994 seminal text by Juan Carlos Garavaglia and Juan Carlos Grosso for the New Spanish context, highlighting their rigorous historical and anthropological approach (and especially a systematic analysis of available census data), which allowed them to argue that ethnic and social groups were not competing categories but mutually significant systems of representation.

KEYWORDS: Mexico, Garavaglia, Grosso, mestizaje, 18th century.

[Recibido: 20/11/19; Aceptado: 5/2/19] 
«Cada concepto fundamental contiene varios estratos profundos procedentes de significados pasados, así como expectativas de futuro de diferente calado» (R. Koselleck, Historia de los conceptos y conceptos de historia, pp. 37-38).

Pocos conceptos presentan un despliegue histórico con múltiples capas de significación acumuladas tan interesantes como el de «mestizo». Es por ello imprescindible, para cualquier aproximación al término, focalizar primeramente el análisis en el contexto histórico que le dio origen y sentido; esto es, la América hispánica colonial y postcolonial. Y a continuación, examinar con detenimiento su fundamento en la idea de mestizaje y su relación con el concepto moderno de «raza». ${ }^{1}$ Este fue el espíritu del grupo AHCISP (Antropologia i Història de la Construcció d'Identitats Socials i Polítiques), auspiciado por el Departament d'Antropologia Social i Cultural de la Universitat Autònoma de Barcelona en 2004. Para los miembros del grupo el mestizaje era —y continúa siendo- un fenómeno sugerente, pues esclarece la lógica y la dinámica de sistemas de clasificación social. La categoría del/a «mestiz@» connota una ubicación e identificación socio-políticas ambiguas. Fruto de la interacción entre individu@s adscriptos a grupos «raciales», étnicos, sociales y/o culturales que se conciben como distintos y excluyentes, 1@s «mestiz@s» provocan intranquilidad y desconfianza pues desafían las categorías sociales establecidas. Pero esta desconfianza ante $1 @_{\mathrm{S}}$ «mestiz@s» revitaliza al mismo tiempo las presuntas marcas diacríticas que sostienen el sistema de clasificación social, ya que las presupone.

El 4 y 5 de diciembre de 2008 AHCISP organizó las Jornadas Internacionales: Identidades Ambivalentes a debate, coordinado por la Dra. Mónica Martínez Mauri (cuya tesis doctoral de 2007 sobre los Kuna Yala de Panamá, leída en la École des Hautes Études en Sciences Sociales, fue codirigida por Juan Carlos Garavaglia). ${ }^{2}$ En la primera sesión de las Jornadas se analizó la cuestión ambivalente del mestizaje en el ámbito estricto de América Latina. La primera ponencia ofreció una relectura de un texto clásico que Juan Carlos Garavaglia, presente en el evento, escribió con Juan Carlos Grosso, «Criollos, mestizos e indos: etnias y clases sociales en México colonial a fines del siglo XVIII», en el que analizaba el mestizaje no tanto como categoría legal sino como el resultado de un proceso lógico de interacción entre grupos étnico-sociales con intereses comunes. ${ }^{3}$

Otros historiadores del mundo mexicano, como John Chance y William B. Taylor, ${ }^{4}$ habían expresado sus dudas acerca de la utilidad de conceptos étnico-raciales para anali-

1. Al respecto, véase Hering Torres, Max S., «Purity of Blood. Problems of Interpretation», y Chaves, M. ${ }^{a}$ Eugenia, «Race and Caste. Other Words and Other Worlds», en Hering Torres, Max S., María Elena Martínez y David Nirenberg (eds.), Race and Blood in the Iberian World, vol. 3, Viena \& Berlin, LIT Verlag, 2012, pp. 1138; 39-58.

2. Martínez Mauri, Mónica, De Tule Nega a Kuna Yala. Mediación, territorio y ecología en Panamá, $1903-$ 2004, Tesis Doctoral, École des Hautes Études en Sciences Sociales, Paris, 2007.

3. Garavaglia, Juan Carlos y Juan Carlos Grosso, «Criollos, mestizos e indios: etnias y clases sociales en México colonial a fines del siglo XVIII». Secuencia, 29, 1994, pp. 39-80.

4. Chance, John K. y William B. Taylor, «Estate and class in a colonial city: Oaxaca in 1792». Comparative Studies in Society and History, 19:4, 1977, pp. 454-487; Chance, John K., Razas y clases de la Oaxaca colonial, Mexico, DF, Instituto Nacional Indigenista, Serie de Antropología Social, n. ${ }^{\circ}$ 64, 1982. 
zar la estratificación social de finales del siglo XVIII. Del mismo modo, historiadores del mundo andino, como Antonio Acosta y Bernard Lavallé, 5 ya habían planteado las mismas consideraciones al analizar los términos «criollo» y «mestizo», en ámbitos geográficos distintos. ${ }^{6}$ El mestizaje no existe como categoría social preexistente, sino más bien como un proceso dinámico caracterizado por las diversas mezclas interétnicas entre blancos, negros e indios, sin excluir otras dinámicas de diferenciación y exclusión. ${ }^{7}$ Con todo, la aleatoriedad de la mezcla nos obliga a repensar nuestras categorías analíticas, así como el reconocimiento de estos espacios intermedios. ${ }^{8}$

Frente a las categorías de clasificación étnico-racial impuestas desde la metrópoli, como la sangre indígena o mestiza, asimiladas a linajes plebeyos o a la ilegitimidad e impureza étnica, los grupos plebeyos fomentaron otros marcadores étnico-sociales que no eran permanentes ni dependían exclusivamente del fenotipo, como los oficios, la vestimenta, la reputación o conducta moral y la lengua, pero que facilitaban la movilidad social. ${ }^{9}$ Dichos marcadores culturales constituyen los elementos centrales en la constitución de los límites étnicos. Si las autoridades metropolitanas insistían en una supuesta clasificación objetiva «desde arriba», la realidad concreta de ejercer un oficio determinado, vestir y hablar de una determinada manera y vivir dentro o fuera de una ciudad consti-

5. Acosta Rodríguez, Antonio, «Conflictos sociales y políticos en el sur peruano (Puno, La Paz, Laicacota, 1660-1668)», en VV.AA., Primeras Jornadas de Andalucía y América. La Rábida, Tomo II, Huelva, Instituto de Estudios Onubenses, 1981, pp. 29-51; Acosta Rodríguez, Antonio, «Sobre criollos y criollismo». Revista Andina, n. ${ }^{\circ}$ 1, 1984, pp. 73-88; Lavallé, Bernard, Recherches sur l'apparition de la conscience créole dans la vice-royauté du Pérou: l'antagonisme hispano-créole dans les ordres religieux (XVI-XVII siècle). Tesis Doctoral, Université de Bordeaux, III. Atelier National de Reproduction de Thèses, Université de Lille, 1982.

6. Para una crítica de la capacidad heurística de la oposición entre peninsulares y criollos, véase Acosta Rodríguez, Antonio, «Conflictos sociales y políticos en el sur peruano...», cit., pp. 29-51; Acosta Rodríguez, Antonio, «Sobre criollos y criollismo»..., cit., pp. 73-88.

7. Como señalan Max S. Hering, M. ${ }^{a}$ Elena Martínez y David Nirenberg, «racial constructs are not uniform or fixed and they rarely work alone. Rather, they are the products of particular cultural traditions and social context and are linked to other social relations, processes or exclusion, and systems of signification»cf. Hering Torres, Max S., «Editorial», en Hering Torres, Max S., Martínez, María Elena y Nirenberg, David (eds.), Race and Blood..., cit., p. 2. Véase también Burns, Kathryn, «Unfixing Race»..., cit., en Gotkowitz, Laura (eds.), Histories of Race and Racism. The Andes and Mesoamerica from Colonial Times to the Present, Durham \& London, Duke University Press, 2011, p. 66.

8. Garavaglia, Juan Carlos y Juan Carlos Grosso, «Criollos, mestizos e indios...», cit., pp. 39-80; Amselle, Jean-Loup, Logiques métisses: anthropologie de l'identité en Afrique et ailleurs, Paris, Payot «Bibliothèque scientifique Payot», 1990; Gruzinski, Serge, El pensamiento mestizo, Barcelona, Paidós, 2000, p. 60; Stolcke, Verena, «El mestizo no nace, se hace», en Verena Stolcke y Alexandre Coello (eds.), Identidades ambivalentes en América (siglos XVI-XXI), Barcelona, Bellaterra, 2008, pp. 17-58; Hering Torres, Max S., «Purity of Blood..., cit., p. 26.

9. Fisher, Andrew B. y Matthew D. O'Hara, «Introduction: Racial Identities and Their Interpreters in Colonial Latin America», en Fisher, Andrew B. y Matthew D. O'Hara, Imperial Subjects. Race and Identity in Colonial Latin America, Durham, Duke University Press, 2009, p. 11. Véase también Graubart, Karen B., «The Creolization of the New World: Local Forms of Identification in Urban Colonial Peru, 1560-1640», Hispanic American Historical Review, 89:3, 2009, pp. 471-499; Presta, Ana María, «Undressing the Coya and Dressing the Indian Woman: Market Economy, Clothing, and Identities in the Colonial Andes, La Plata (Charcas), Late Sixteenth and Early Seventeenth Centuries», Hispanic American Historical Review, 90:1, 2009, pp. 41-53. 
tuían percepciones concretas que los grupos plebeyos tenían de su posición en la sociedad colonial.

Por este motivo, los historiadores y etnohistoriadores coloniales, como Antonio Acosta, Bernard Lavallé, William B. Taylor, Magnus Mörner, Juan Carlos Garavaglia, Serge Gruzinski y Ana María Presta, entre otros, expresaron sus dudas acerca de la operatividad de tales categorías analíticas, avisando que de no revisarlas a fondo podrían distorsionar fuertemente los análisis sociales. En concreto, Garavaglia y Grosso, siguiendo las investigaciones de antropólogos africanistas, como Jean-Loup Amselle, ${ }^{10}$ subrayaron no con poca sorna que «si no cambiamos el enfoque del asunto, corremos el riesgo de caer en una discusión similar a la del sexo de los ángeles (...) fundamentalmente porque en América colonial, los límites étnicos y los límites sociales nunca pueden tomarse como categorías contrapuestas, sino como sistemas de representación mutuamente significativos». ${ }^{11}$ ¿Cuál era la diferencia entre un «criollo», un «mestizo» y un «español»? ¿El «criollo» se consideraba a sí mismo como tal, o por el contrario, fue «inventado» por las elites locales o peninsulares? ¿Esos términos significaban lo mismo en los virreinatos de México y el Perú, en Lima que en las tierras altas del Cuzco, en Veracruz que en Acapulco, en Puebla, Tepeaca o en Oaxaca?

En 1987 el INAH publicó una obra que resultó clave en la constitución de la etnohistoria como disciplina o método de la antropología o de la historia: La etnohistoria en Mesoamérica y los Andes, compilado por Juan Manuel Pérez Cevallos y José Antonio Pérez Gollán. Los artículos de Carlos Martínez Marín, ${ }^{12}$ así como las reflexiones de Juan Manuel Pérez Zevallos en este y otros trabajos ${ }^{13}$ sirvieron para definir la etnohistoria como la disciplina dedicada al estudio de las sociedades autóctonas que sufrieron — de manera muy distinta y fragmentada para cada región- la dominación colonial. ${ }^{14} \mathrm{El}$ uso de fuentes documentales introdujo a los antropólogos mexicanos en los métodos del historiador, y al mismo tiempo, permitió a los historiadores mexicanos incorporar una visión antropológica centrada en la diversidad cultural. ${ }^{15}$ En este sentido, México profundo. Una civilización negada de Guillermo Bonfil, ${ }^{16}$ representó una vuelta de tuerca al papel que correspondía a indios y campesinos en la modernización económica del país. ${ }^{17}$ Influido

10. Amselle, Jean-Loup, Logiques métisses... cit.

11. Ibidem, citado en Garavaglia, Juan Carlos, y Juan Carlos Grosso, «Criollos, mestizos e indios...», cit., p. 40.

12. Martínez Marín, Carlos, «La etnohistoria», en Juan Manuel Pérez Cevallos y José Antonio Pérez Gollán (comp.), La etnohistoria en Mesoamérica y los Andes, México, DF: Instituto Nacional de Antropología e Historia, 1987.

13. Ibidem y Pérez Zevallos, «La etnohistoria en México», Desacatos, n. ${ }^{\circ} 7,2001$, pp. 103-110.

14. Menegus, Margarita, Los indios en la historia de México, México, Centro de investigación y docencia económicas \& Fondo de Cultura Económica, 2006, p. 59.

15. Un trabajo representativo de este alcance histórico-antropológico sobre el proceso de cambio y asimilación a la estructura colonial de la sociedad maya-yucateca fue el trabajo de Farris, Nancy M., Maya Society under Colonial Rule. The Collective Entreprise of Survival, Princeton, Princeton University Press, 1984.

16. Bonfil, Guillermo, México profundo. Una civilización negada, México, Grijalbo, 1987.

17. En un artículo de 1972, Guillermo Bonfil Batalla denunciaba la categoría de «indio» como producto 
por las tesis de James Scott ${ }^{18}$ sobre las formas de resistencia campesina, Bonfil abogaba por la necesidad del control de los indios campesinos de su propia cultura, creando una separación entre dos mundos: por un lado, el «México imaginario» de los grupos dominantes; y por el otro, el «México profundo» de los grupos en resistencia. Criticaba el indigenismo oficial del PRI, considerado como una superestructura impuesta (un «discurso público», que diría Scott) sobre el México real, y en su lugar reivindicaba la existencia de una civilización mesoamericana que constituía una matriz civilizatoria —algo así estaba implícito en el análisis y reconstrucción que hizo Enrique Florescano ${ }^{19}$ de la memoria colectiva mexicana - a partir de la cual se podía conseguir la integración de la población autóctona del país. ${ }^{20}$ El principal reto de la antropología, según Bonfil, era escribir la historia de los pueblos autóctonos y de otros sectores sociales excluidos y negados de la academia mexicana. Reivindicar la perspectiva del actor (indígena) suponía no sólo un cuestionamiento de las condiciones de desigualdad y diferencia sino recuperar la historicidad del sujeto social y analizar las acciones micro-grupales o comunitarias. ${ }^{21}$

En los años noventa el Instituto Nacional Indigenista (INI) protagonizó un giro multicultural, enfatizando el carácter multiétnico de la nación mexicana, así como el derecho a la diversidad cultural de los pueblos. La reforma constitucional de 1992 abrió un nuevo espacio de lucha y la negociación por los derechos indígenas, lo que permitió al INI convertirse en una agencia estratégica. Esto quedó patente en 1994, tras la revuelta zapatista del estado sureño de Chiapas, cuando el INI amplió y fortaleció sus programas en defensa de la autonomía indígena, del control de sus propios recursos, de sus tierras y formas de conocimiento. 22

Ese mismo año, Juan Carlos Garavaglia y Juan Carlos Grosso publicaban el artículo que comentamos. El enfoque histórico y antropológico riguroso (y en especial un análisis sistemático de los datos censales disponibles), les permitió plantear que los grupos étnicos y sociales no eran categorías contrapuestas sino sistemas de representación mutua-

de la dominación colonial. $C f$. Bonfil Batalla, Guillermo, «El concepto de indio en América: una categoría de la situación colonial», Anales de Antropología (México), 9, 1972, pp. 105-124.

18. Scott, James C., Weapons of the Weak. Everyday Forms of Peasant Resistance, New Haven, Yale University Press, 1985.

19. Florescano, Enrique, Memoria indígena, Madrid, Taurus, 1999.

20. De la Peña, Guillermo, «Apuntes sobre la antropología sociocultural en México», en Díez Hurtado, Alejandro (ed.), La antropología ante el Perú de hoy. Balances regionales y antropologías latinoamericanas, Lima, Pontificia Universidad Católica del Perú (PUCP) \& CISEPA, p. 172. Igualmente, El pasado indígena, México, Fondo de Cultura Económica, 2001, de Alfredo López Austin y Leonardo López Luján, denunciaba las políticas neoliberales que habían sumido a los pueblos indígenas en la miseria. Las armas de la resistencia no eran muchas, pero entre las más valiosas, según los autores, existe «un legado cultural que, forjado a lo largo de 13 siglos, durante todo el Preclásico Temprano, formó la esencia de Mesoamérica», López Austin, Alfredo y Leonardo López Luján, El pasado indígena, México, Fondo de Cultura Económica, 2001, p. 306.

21. Bonfil Batalla, Guillermo, «Historias que no son todavía historia», en Pereyra, Carlos, Historia para qué. México, Siglo XXI [1980] 2009, pp. 229-233. Véase también Bonfil Batalla, Guillermo, «Del indigenismo de la revolución a la antropología crítica», en Warman, A. (eds.), De eso que llaman antropología mexicana, México, Nuevo Tiempo, 1970, pp. 39-65.

22. De la Peña, Guillermo, «Apuntes sobre...», cit., p. 180. 
mente significativos. En un intento de aclarar los términos del problema clasificatorio ambos sugirieron analizar el mestizaje no tanto como cuestión exclusivamente jurídica, sino como el resultado de un proceso lógico de interacción entre grupos étnico-sociales con intereses (políticos, económicos) compartidos. A menudo estas categorías socio-étnicas — «mestizos», «criollos», «castizos» (mezcla de español con mestizo), «mulatos», etc.- habían sido entendidas como un intento de establecer los límites étnico-raciales de grupo a que se ajustaban los individuos. En cambio, la propuesta de los autores consistió en examinar otros elementos de clasificación social, como los oficios que ejercen, los lugares de residencia y las formas particulares de vestir o hablar, resaltando en especial los factores dinámicos que daban lugar a nuevos contextos culturales. Es decir, no se trataba de categorías inmanentes o «naturales» sino sociales que no podían entenderse al margen de fenómenos que son históricos, complejos y heterogéneos. Precisamente el hecho de enfocar sus estudios en el ámbito urbano, considerado como un «espacio ideal para los encuentros entre grupos socialmente diversos», permitió a Garavaglia y Grosso proporcionar una visión más dinámica, fluida y relacional de la vida cotidiana de los grupos sociales que la imagen tradicional que se tenía de las jerarquías socio-políticas en la América colonial. ${ }^{23} \mathrm{El}$ texto introdujo una nueva perspectiva a la discusión sobre el mestizaje que ponía en entredicho la concepción estática y estrictamente vinculada a la cuestión étnica (incluso de lectura esencialista) de las relaciones entre los grupos sociales en juego, enfatizando la «auto-definición» del grupo en cuestión. ${ }^{24}$

Parte del problema analítico reside en la utilización que se había hecho del término mestizaje. Para Garavaglia y Grosso, no existe un mestizaje, sino varios. ${ }^{25}$ Es evidente que las diversas «mezclas» entre la población blanca, negra e india que resultaron de los matrimonios interétnicos eran solo la punta del iceberg de un proceso social mucho más amplio que incluía las uniones libres, el concubinato y la bigamia. La frecuencia de las uniones extra-matrimoniales más o menos inestables y de la ilegitimidad a lo largo del siglo XVII en Hispano-América no podía explicarse exclusivamente por los abusos sexuales ejercidos por los conquistadores y sus descendientes, sino que había que tener en cuenta también la influencia reciproca de los valores sociales y culturales entre los grupos. ${ }^{26}$ A finales del siglo XVII, las pautas de emparejamiento y reproducción se fueron modificando a causa de la contigüidad cultural y social de los «grupos plebeyos», ${ }^{27}$ favoreciendo las relaciones necesarias que permitieron los intercambios — no solo sexuales,

23. Garavaglia, Juan Carlos y Juan Carlos Grosso, «Criollos, mestizos e indios...», cit., p. 67.

24. En este sentido, más que ubicar a los individuos en categorías supuestamente objetivas que no existen en la realidad, lo que plantean Garavaglia y Grosso es profundizar en un análisis de un «continuum de auto y endoclasificación», Ibidem, p. 46.

25. Ibidem, p. 42.

26. Ibidem, p. 43.

27. En el siglo XVIII los «grupos plebeyos» se caracterizaban no tanto por su categoría étnica como por su baja condición social. Véase Viqueira Albán, Juan Pedro, Propriety and Permissiveness in Bourbon Mexico, Wilmington, Delaware, SR Book [1987] 1999, p. XVII; R. Cope, Douglas, The Limits of Racial Domination. Plebeian Society in Colonial Mexico, 1660-1720, Wisconsin, Madison, Wisconsin University Press, 1994. 
sino de todo tipo- entre individuos. En otras palabras, el «mestizaje» sería el mecanismo social que posibilitaba una eventual relación sexual, fruto de la cual podría aparecer un hijo «mestizo». ${ }^{28}$

En la sociedad colonial hispano-americana, la familia consagrada por los sacramentos se convirtió en una de las principales preocupaciones de las autoridades civiles y eclesiásticas. A lo largo del siglo XVIII los matrimonios entre «españoles» e indias aumentaron, pero también los nacimientos ilegítimos, fruto del elevado numero de uniones libres -o concubinatos. ${ }^{29}$ Esto se explica, en parte, porque por razones socio-económicas y culturales el concubinato no era extraño a la sociedad española. Las leyes españolas permitían las uniones sexuales no formalizadas. Sin embargo, a partir del Concilio de Trento (154563) se prohibieron algunas formas de cohabitación entre personas sin ningún impedimento para casarse, como la barraganía, definida como un «vínculo contractual entre dos personas solteras, que de mutuo acuerdo establecían una «compañía de mesa y cama» —como solía llamarse- y que, a diferencia del vínculo matrimonial, podía disolverse también por mutuo acuerdo». ${ }^{30}$

Aparte de la desigualdad social entre los integrantes de las parejas, otra razón podría ser que muchos de estos plebeyos ya estaban casados. Contraer otro matrimonio conllevaba el riesgo de ser acusado de bigamia. La iglesia, siempre vigilante, obligaba a los amancebados a casarse, lo que a veces suponía un aumento de bígamos. Los indios acusados del delito de «dúplice matrimonio» eran juzgados por los tribunales eclesiásticos, mientras que los españoles tenían que vérselas con los fiscales inquisitoriales del Santo Oficio. $^{31}$

La existencia de unas zonas de contacto social entre grupos sociales distintos que interactúan y negocian constantemente es un aspecto a resaltar en la determinación de fronteras étnicas. Como ya señaló Frederick Barth, ${ }^{32}$ los grupos sociales se definen por su relación con otros grupos. Los datos que proporciona el censo de 1791 de Tepeaca en Puebla, México, contienen información interesante sobre criterios de definición social

28. Garavaglia, Juan Carlos y Juan Carlos, Grosso, «Criollos, mestizos e indios...», cit., p. 44.

29. En su tesis doctoral de 1978, Dennis Nodin Valdés afirmaba que en el siglo XVIII, los españoles de la capital de México vivían en concubinato con las españolas tanto como con las llamadas «castas», D. N. Valdés, 1978, citado en Kuznesof, Elizabeth A., «Raza, clase y matrimonio en la Nueva España: estado actual del debate», en Gonzalvo Aizpuru, Pilar (coord.), Familias novohispanas. Siglos XVI al XIX, Seminario de Historia de la Familia, Centro de Estudios Históricos, México, El Colegio de México, 1991, p. 386.

30. Kuznesof, E. A., «Raza, clase y matrimonio en la Nueva España: estado actual del debate»..., cit., pp. 376-377; Ares Queija, Berta, «Un borracho de chicha y vino». La construcción social del mestizo (Perú, siglo XVI), en Gregorio Salinero (coord.), Mezclado y sospechoso. Movilidad e identidades, España y América (siglos XVI-XVIII). Coloquio Internacional (29-31 de mayo de 2000), Madrid, Colección de la Casa de Velásquez, vol. n. ${ }^{\circ}$ 90, 2005, pp. 122-123.

31. Boyer, Richard, Lives of the Bigamist. Marriage, Family and Community in Colonial Mexico, Albuquerque, University of New Mexico Press, 1995, pp. 13-32.

32. Barth, Fredrik, «Les groupes ethniques et leurs frontières», en Ph. Poutignat et J. Streiff-Fenart (eds.), Théories de l'ethnicité, suivi de Les groupes ethniques et leurs frontières de F. Barth, Paris, PUF [1969] 1995, pp. 203-249; Barth, F., Los grupos étnicos y sus fronteras. La organización social de las diferencias culturales, México, Fondo de Cultura Económica [1969] 1976. 
— parentesco, residencia, afinidad ocupacional y etnicidad — de las familias de la «gente común». Entre 1777 y 1792, por ejemplo, los que se definían como «españoles» aumentaron un $50 \%$, mientras que los «mestizos» y «castizos» lo hicieron solamente un $28 \%$, lo que indica un proceso de blanqueamiento gradual de la población..$^{33}$ Otros autores, como Elizabeth A. Kuznesof, señalaron que los hijos de españoles e indígenas, mestizas o castizas, fueron integrados al grupo dominante, mientras que los otros «grupos mezclados» o «castas» — «castizos» (mezcla de español y mestizo), «moriscos» (mezcla de mulato con español), «mestizos», «mulatos»- fueron relegados por su inferior «calidad»o «condición». ${ }^{34}$

Una sociedad (colonial) que era consciente de su propia fragilidad debía reflejar la jerarquía racial y socioeconómica de sus miembros según la proporción de «sangre española». ${ }^{35}$ Como ya señaló R. Douglas Cope, «all elites were Spaniards, but not all Spaniards were members of the elite». ${ }^{36}$ La sangre transmitía no solamente la «calidad» de las personas sino también sus «defectos» («raza» o mácula) que heredaba su progenie. ${ }^{37} \mathrm{~A}$ finales del siglo XVII, la doctrina de la «pureza de sangre» se asoció a la blancura de piel de los españoles y sus descendientes, mientras que los individuos de color — pardos, negros, bermejos, morenos, loros, leonados, es decir, todos aquellos «no blancos»— eran portadores de una mácula, o «raza» que los convertía en inferiores. ${ }^{38}$

Las primeras pinturas o «cuadros de castas», realizadas por Juan Rodríguez Fonseca (1715) en la Nueva España, respondían a los afanes clasificatorios propios del cientifismo del siglo XVIII. ${ }^{39}$ Aunque las «castas» no fueron registradas como tales en la documentación colonial, sin duda representaron el reflejo de las nuevas generaciones de «cuerpos mestizos» descendientes de padres de fenotipo diferente — «lobos» (mezcla de mulatos con indios), «coyotes» (mezcla de indio con mestizo), «castizos» y «moriscos», entre otras- que proliferaban en los Virreinatos del Perú y de la Nueva España. ${ }^{40}$ Si los histo-

33. Garavaglia, Juan Carlos y Juan Carlos, Grosso, «Criollos, mestizos e indios...», cit., p. 51.

34. Kuznesof, Elizabeth A., «Ethnic and Gender Influences on «Spanish» Creole Society», Colonial Latin American Review, 4:1, 1995, pp. 168-170.

35. Cope, R. Douglas, The Limits of ..., cit., p. 24.

36. Ibidem, p. 25.

37. Hering Torres, Max. S., «Purity of Blood...», cit., p. 24.

38. Ibidem, p. 25; Thomson, Sinclair, «Was There Race in Colonial Latin America? Identifying Selves and Others in the Insurgent Andes», en Gotkowitz, Laura (ed.), Histories of Race and Racism. The Andes and Mesoamerica from Colonial Times to the Present, Durham \& London, Duke University Press, 2011, p. 87.

39. Katzew, Ilona, New World Order. Casta Paintings and Colonial America. New York, Americas Society Art Gallery, 1996; Carrera, Magali M., Imagining Identity in New Spain. Race, Lineage, and the Colonial Body in Portraiture and Casta Paintings, Austin, Texas, University of Texas Press, 2003, p. 88; Katzew, Ilona, Casta Paintings. Images of race in eighteenth-century Mexico. New Haven and London, Yale University Press, 2004.

40. Como es sabido, la Nueva España fue uno de los primeros lugares donde convivieron españoles, esclavos negros e indígenas. En 1521-34, había 8.000 esclavos en Ciudad de México. A lo largo de los siglos XVI y XVII se produjo un comercio sexual, creando nuevos fenotipos, nuevos «cuerpos mestizos». Las razas no existen, pero sí las características fenotípicas contenidas en los códigos genéticos. Así, en el siglo XVII aparecieron los términos populares que se fijarán en la producción administrativa para «racializar» dichos fenotipos. Cf. Peter Wade, citado en Baerga, María del Carmen, Negociaciones de sangre: dinámicas racializantes en el Puerto Rico decimonónico, Madrid, Iberoamericana \& Vervuert \& Ediciones Callejón \& Universidad de 
riadores habitualmente habían leído las «castas» como aquellos «grupos mezclados» que desordenaban las tres categorías del Nuevo Mundo (español, negro, indio), las pinturas de «castas» reordenaban la jerarquía racial, vigente en el periodo colonial, donde los orígenes étnicos y la apariencia física determinaban la posición, el prestigio y los derechos de la persona («pigmentocracia»). ${ }^{41}$ Se trataba, pues, de poner un orden, no en el desorden racial, sino en el desorden terminológico. ${ }^{42}$ Una lógica que, como veremos, no pretendía sino defender la preeeminencia de las élites blancas y sus descendientes, como representantes de la civilización europea, sobre la base del «color», la «calidad» y la raza. ${ }^{43}$

En efecto, el objetivo de la Pragmática Sanción para Evitar Matrimonios y el Abuso de Contraer Matrimonios Desiguales, de Carlos III, sancionada en 1776 y aplicada en 1778 en la América española, consistía en proteger la jerarquía social y racial de las elites hispano-criollas al proporcionar al pater familias un mayor control sobre las opciones matrimoniales de sus hijos, asegurando así la endogamia de clase socio-racial. ${ }^{44} \mathrm{El}$ matrimonio se convirtió, pues, en «un proceso que producía o transformaba identidades raciales». ${ }^{45}$ Para las familias de la gente común (es decir, los «pobres») de Tepeaca lo importante no era tanto la categoría social en sí, como el peligro de convertirse en «pardos» o «indios tributarios».46 La limpieza de sangre se había secularizado (aunque no completamente) para estigmatizar la mezcla con indios, pero sobre todo con africanos o su progenie mulata (las llamadas «castas pardas») que podía «contaminar» a familias de cristianos viejos (peninsulares, «criollos») si no se establecía un severo control genealógico. ${ }^{47}$ Los africanos eran descendientes de Cam y gentes portadoras del estigma de la esclavitud a causa de la maldición de Noé. Debido a su origen incierto se hacía imposible trazar la genealo-

Puerto Rico, 2015, pp. 27-28. Por ejemplo, morisco, en la Nueva España, hace referencia al color de la piel, y por tanto, su significado varió respecto al que tenía originalmente en la Vieja España, cf. Zúñiga, Jean-Paul, «Cuerpos mestizos: genealogía y apariencia en la América hispánica». Ponencia presentada en la Jornada Internacional, El poder dels sistemes de classificació social. Sala de Juntes, Facultat de Filosofia i Lletres, Universitat Autònoma de Barcelona (UAB), 27 de octubre de 2011).

41. Castro Morales, Efraín, «Los cuadros de Castas de la Nueva España», Jabrbuch für Geschichte von Staat, Wirtschaft, und Gesellschaft Lateinamerikas, 20, 1983, pp. 671-690. Para la construcción del «color como maleficio», en las Antillas francesas, véase Bonniol, Jean-Luc, La couleur comme maléfice, Paris, Albin Michel, 1992.

42. Como señala Jean-Paul Zúñiga, existía una gran variedad regional de nombres y «etiquetas raciales» que se aplicaban de manera variada según la región. Por esta razón era necesario dar cuerpo intelectual a la realidad colonial por medio de taxonomías claras sobre una realidad caótica y variada, $c f$. Zúñiga, Jean-Paul, «Cuerpos mestizos: genealogía y..., cit.

43. Hering Torres, Max, S., «Purity of Blood...», cit., p. 29. Para un reciente análisis del «sistema de castas» y sus limitaciones, véase el reciente trabajo de Vinson, Ben III, Before Mestizaje: The Frontiers of Race and Caste in Colonial Mexico, Cambridge, Cambridge University Press, 2017.

44. Significativamente, «mulatos, negros, coyotes y personas de castas y razas similares» quedaban exentos de dicha Pragmática porque ninguno de ellos poseía honores sociales que proteger de un matrimonio desigual: Stolcke, Verena, «Los mestizos no nacen, se hacen»..., cit., pp. 49-50.

45. Baerga, María del Carmen, Negociaciones de sangre..., cit., p. 18.

46. Garavaglia, Juan Carlos y Juan Carlos Grosso, «Criollos, mestizos e indios...», cit., p. 47.

47. Martínez, M. ${ }^{a}$ Elena, Genealogical Fictions. Limpieza de sangre, Religion, and Gender in Colonial Mexico, Stanford, Stanford University Press, 2008, p. 248. 
gía de su fe, «ya que, en muchos casos, la esclavitud cercenaba las relaciones de parentesco, lo que imposibilitaba la identificación de los ancestros». ${ }^{48}$ Por este motivo se hacía necesario fiscalizar los enlaces matrimoniales con el fin de preservar la «calidad» de los contrayentes. ${ }^{49}$ Prueba de ello es el edicto que la Audiencia de México añadió a la Pragmática Sanción en 1781 en el que se especificaba que «los mestizos descendientes de españoles y mujeres indias o a la inversa, así como los castizos, merecen ser distinguidos del resto de razas [...] y están igualmente sujetos a los requerimientos y demandas que prescribe la Pragmática Real». ${ }^{50}$ El edicto evidenciaba la preocupación por la endogamia socio-racial de clase que estaba siendo amenazada por el aumento de alianzas matrimoniales entre personas «desiguales» dentro de la jerarquía social. ${ }^{51}$

Otro ejemplo es el de la escasa correlación existente entre categorías sociales y actividades ocupacionales. El caso de los ahora definidos como «criollos» (es decir, los descendientes de los primeros españoles nacidos en territorio americano) es especialmente significativo, pues a finales del siglo XVIII se ocupaban de unos oficios — artesanía- que parecerían estar reservados a otros sectores considerados «racialmente» inferiores. ${ }^{52} \mathrm{En}$ la ciudad minera de Guanajuato, comenta David Brading, también realizaban todo tipo de trabajos, sobrepasando a mestizos y mulatos. ${ }^{53}$

A comienzos del siglo XVIII, los Virreinatos americanos estaban integrados, además de la población indígena, por una población mayoritariamente criolla. Estos «criollos» comprendían tanto a los hijos de los europeos de primera generación como a aquellos individuos mezclados con indios, mulatos o negros que podrían ser incluidos en la categoría más general de «castas». A diferencia de la rigidez de las castas de la India, estos grupos de diferente «condición», «calidad», o «clase» alternaban sus posiciones en lo que Ben Vinson III ha recientemente definido como «cast pluralism». ${ }^{54}$ Es indudable que aquellos «hijos de la tierra» no constituían un grupo de ribetes definidos, resultando difícil discernir el modo en que estas personas encauzaron sus intereses dentro de la sociedad en que vivían mediante la delimitación de los márgenes sociales.

Sea como fuere, algunos burócratas españoles, como Antonio de Ulloa (1716-95), no

48. Baerga, M. ${ }^{a}$ del Carmen, Negoaciaciones de sangre..., cit., p. 170.

49. En el siglo XVIII se pensaba que «mulatos, pardos, zambos y otras castas estaban viciados desde su nacimiento y tenían malos hábitos, siendo la mayoría de ellos espurios, adúlteros e ilegítimos» cf. Konetzske, Richard, Colección de Documentos para la Historia de la Formación Social de Hispanoamérica, 1493-1810. vol. III, Madrid, CSIC, 1962, pp. 823-824. Para el caso portorriqueño del siglo XIX, véase Baerga, M. ${ }^{a}$ del Carmen, Negoaciaciones de sangre..., cit., pp. 155-196.

50. Konetzske, Richard, citado en Stolcke, Verena, «Los mestizos no nacen, se hacen...», cit., p. 50.

51. Marre, Diana, «Historia de la familia e historia social. La aplicación de la Pragmática Sanción de Carlos III en América Latina: una revisión». Quaderns de l'Institut Català d'Antropologia, 10, pp. 223-230.

52. Garavaglia, Juan Carlos y Juan Carlos Grosso, «Criollos, mestizos e indios...», cit., p. 51.

53. Brading, David, Mineros y comerciantes en el México borbónico, 1763-1810, México, Fondo de Cultura Económica, 1975, pp. 349-401.

54. History Hub. Episode 18. Vinson, Ben III - Mestizaje and the Frontiers of Race and Caste in Colonial Mexico. <https://player.fm/series/kingdom-empire-and-plus-ultra-conversations-on-the-history-of-portugaland-spain-1415-1898/ep18-ben-vinson-mestizaje-and-the-frontiers-of-race-and-caste-in-colonial-mexico > . 
disimulaban su desprecio hacia los que se auto-definían como «criollos», a quienes consideraban degradados por el ambiente americano, la alimentación, y sobre todo, por la mezcla con individuos de «inferior calidad» -indios y negros. ${ }^{55}$ Ciertamente, el aumento de los «criollos» confirmaba que los individuos adaptaban las categorías étnicas a sus necesidades vitales. La autodefinición étnica cobró importancia en relación con los «otros», es decir, en la medida en que ciertas clasificaciones («pardo», «negro», «mulato», «indio») impedían o dificultaban el ascenso social. Hablar de «razas» o clases económicamente objetivas no tiene mucho sentido. En su lugar, Garavaglia y Grosso proponían definir las adscripciones sociales a partir de otras variables, como el fenotipo, la lengua, la ocupación, la residencia o el nivel de educación e integración en los valores de la cultura hegemónica.

En resumen, esta visión dinámica del mestizaje entre la «gente común» de Tepeaca de finales del siglo XVIII nos muestra un complejo sistema de representaciones sociales en el que los criterios de definición étnico-social (propio o ajeno) derivan de un conjunto complejo de variables que van más allá de la «tiranía de la mezcla». Autores como Tamar Herzog sostienen que las prácticas de exclusión no pueden explicarse únicamente a partir de sistemas de clasificación basados en la «raza», sino en otros discursos basados en nociones weberianas de pertenencia y no pertenencia a comunidades políticas locales. ${ }^{56}$ Categorías legales como «vecinos» $\mathrm{y}$ «naturales» podían aplicarse indistintamente a españoles, «criollos», «mestizos» e indios, independientemente de su carácter étnico, frente a los que se consideraban «extranjeros». Otros estudios, como el de Peter B. Villella, han puesto de manifiesto el carácter «inventado» de las categorías de «indio» y «mestizo», en el México central de los siglos XVII-XVIII. ${ }^{57}$

En definitiva, el estudio de caso de Garavaglia y Grosso constituyó un ejemplo pionero de cómo la búsqueda y el examen innovador de los datos permiten descubrir el mestizaje como resultado de interrelaciones sociales previas que hacen posible el encuentro y el emparejamiento más que como la consecuencia de diferencias étnico-sociales pre-existentes. Sus análisis, aunque estrictamente confinados a los datos empíricos obtenidos en un marco histórico y geográfico muy específico, permiten, además, desplegar una reflexión general sobre el propio concepto de mestizaje que va más allá del escenario hispano-americano. Al documentar que los movimientos de población y de interrelaciones, identidades e identificaciones, son una realidad en el México colonial, nos revelan como estos procesos de interacción entre grupos sociales definidos como diferentes no siempre generan los mestizajes étnicos o culturales que a veces se pretenden, desdeñando

55. Earle, Rebecca, The Body of the Conquistador: Food, Race and the Colonial Experience in Spanish America, 1492-1700, Cambridge, Cambridge University Press, 2013.

56. Herzog, Tamar, «Beyond Race: Exclusion in Early Modern Spain and Spanish America», en Hering Torres, Max S., María Elena Martínez y David Nirenberg (eds.), Race and Blood..., cit., pp. 153-156. Véase también Laura Gotkowitz, «Introduction: Racisms of the Present and the Past in Latin America», en Gotkowitz, Laura (ed.), Histories of Race..., cit., pp. 9-10.

57. Villella, Peter B., Indigenous Elites and Creole Identity in Colonial Mexico, 1500-1800, New York, Cambridge University Press, 2016. 
las circunstancias socio-simbólicas y estructurales locales concretas. El trabajo de Garavaglia y Grosso nos remite a anteriores espacios de encuentro entre grupos sociales contrapuestos, de yuxtaposición de culturas hegemónicas y populares, de tensión entre puntos de intercambio y puntos de discriminación. Estos mestizajes nos invitan a continuar pensando y investigando el devenir de categorías sociales múltiples, híbridas o mezcladas, en distintos contextos históricos y sociopolíticos, que trascienden el «mestiz@» colonial hispano-american@.

\section{Bibliografía}

Acosta Rodríguez, Antonio, «Conflictos sociales y políticos en el sur peruano (Puno, La Paz, Laicacota, 1660-1668)», en VV.AA., Primeras Jornadas de Andalucía y América. La Rábida, tomo II, Huelva, Instituto de Estudios Onubenses, 1981, pp. 29-51.

—, «Sobre criollos y criollismo». Revista Andina, núm. 1, 1984, pp. 73-88.

Amselle, Jean-Loup, Logiques métisses: anthropologie de l'identité en Afrique et ailleurs, Paris, Payot «Bibliothèque scientifique Payot», 1990.

Ares Queija, Berta, «"Un borracho de chicha y vino”. La construcción social del mestizo (Perú, siglo XVI)», Salinero, Gregorio (coord.), Mezclado y sospechoso. Movilidad e identidades, España y América (siglos XVI-XVIII), coloquio Internacional (29-31 de mayo de 2000), Madrid, Colección de la Casa de Velázquez, vol. 90, 2005, pp. 122-123.

Baerga, María del Carmen. Negociaciones de sangre: dinámicas racializantes en el Puerto Rico decimonónico, Madrid, Iberoamericana \& Vervuert \& Ediciones Callejón \& Universidad de Puerto Rico, 2015.

Barth, Fredrik, «Les groupes ethniques et leurs frontières», en Ph. Poutignat et J. Streiff-Fenart (eds.), Théories de l'ethnicité, suivi de Les groupes ethniques et leurs frontières de F. Barth, Paris, PUF [1969] 1995, pp. 203-249.

-, Los grupos étnicos y sus fronteras. La organización social de las diferencias culturales, México, Fondo de Cultura Económica [1969] 1976.

Bonfil Batalla, Guillermo, «Del indigenismo de la revolución a la antropología crítica», en Warman, A. (eds.), De eso que llaman antropología mexicana, México, Nuevo Tiempo, 1970, pp. 39-65.

-, «El concepto de indio en América: una categoría de la situación colonial», Anales de Antropología (México), núm. 9, 1972, pp. 105-124.

—, México profundo. Una civilización negada, México, Grijalbo, 1987.

—, «Historias que no son todavía historia», en Pereyra, Carlos, Historia para qué. México, Siglo XXI [1980] 2009, pp. 229-233.

Bonniol, Jean-Luc, La couleur comme maléfice, Paris, Albin Michel, 1992.

Boyer, Richard, Lives of the Bigamist. Marriage, Family and Community in Colonial Mexico, Albuquerque, University of New Mexico Press, 1995.

Brading, David, Mineros y comerciantes en el México borbónico, 1763-1810, México, Fondo de Cultura Económica, 1975.

Burns Kathryn, «Unfixing Race», en Gotkowitz, Laura (ed.), Histories of Race and Racism. The Andes and Mesoamerica from Colonial Times to the Present, Durham \& London, Duke University Press, 2011, pp. 57-71. 
Carrera, Magali M., Imagining Identity in New Spain. Race, Lineage, and the Colonial Body in Portraiture and Casta Paintings, Austin, Texas, University of Texas Press, 2003.

Castro Morales, Efraín, «Los cuadros de Castas de la Nueva España», Jabrbuch für Geschichte von Staat, Wirtschaft, und Gesellschaft Lateinamerikas, núm. 20, 1983, pp. 671-690.

Chance, John K., Razas y clases de la Oaxaca colonial, Mexico City, Instituto Nacional Indigenista, Serie de Antropología Social, n. ${ }^{\circ}$ 64, 1982.

Chance, John K. y William B. Taylor, «Estate and class in a colonial city: Oaxaca in 1792», Comparative Studies in Society and History, vol. 19, n. ${ }^{\circ}$ 4, 1977, pp. 454-487.

Chaves, M. ${ }^{a}$ Eugenia, «Race and Caste. Other Words and Other Worlds», en Hering Torres, Max S., Martínez, María Elena y Nirenberg, David (eds.), Race and Blood in the Iberian World, vol. 3, Viena \& Berlin, LIT Verlag, 2012, pp. 39-58.

Cope, R. Douglas, The Limits of Racial Domination. Plebeian Society in Colonial Mexico City, 16601720, Madison, Wisconsin, University of Wiscosin Press, 1994.

De la Peña, Guillermo, «Apuntes sobre la antropología sociocultural en México», en Díez Hurtado, Alejandro (ed.), La antropología ante el Perú de hoy. Balances regionales y antropologías latinoamericanas, Lima, Pontificia Universidad Católica del Perú \& CISEPA, 2008, pp. 166-180.

Earle, Rebecca, The Body of the Conquistador: Food, Race and the Colonial Experience in Spanish America, 1492-1700, Cambridge, Cambridge University Press, 2013.

Farris, Nancy M., Maya Society under Colonial Rule. The Collective Entreprise of Survival, Princeton, Princeton University Press, 1984.

Fisher, Andrew B. y Matthew D. O’Hara, «Introduction: Racial Identities and Their Interpreters in Colonial Latin America», en Fisher, Andrew B. y Matthew D. O'Hara, Imperial Subjects. Race and Identity in Colonial Latin America, Durham, Duke University Press, 2009.

Florescano, Enrique, Memoria indígena, Madrid, Taurus, 1999.

Garavaglia, Juan Carlos y Grosso, Juan Carlos, «Criollos, mestizos e indios: etnias y clases sociales en México colonial a fines del siglo XVIII», Secuencia, núm. 29, 1994, pp. 39-80.

Gotkowitz, Laura, «Introduction: Racisms of the Present and the Past in Latin America», en Gotkowitz, Laura (ed.), Histories of Race and Racism. The Andes and Mesoamerica from Colonial Times to the Present, Durham \& London, Duke University Press, 2011, pp. 1-39.

Graubart, Karen B., «The Creolization of the New World: Local Forms of Identification in Urban Colonial Peru, 1560-1640», Hispanic American Historical Review, vol. 89, n. ${ }^{\circ} 3$, 2009, pp. 471 499.

Gruzinski, Serge, El pensamiento mestizo, Barcelona, Paidós, 2000.

Hering Torres, Max. S., «Purity of Blood. Problems of Interpretation», en Hering Torres, Max S., María Elena Martínez y David Nirenberg (ed.), Race and Blood in the Iberian World, vol. 3, Viena \& Berlin, LIT Verlag, 2012, pp. 11-38.

Herzog, Tamar, «Beyond Race: Exclusion in Early Modern Spain and Spanish America», en Hering Torres, Max S., Martínez, María Elena y Nirenberg, David (eds.), Race and Blood in the Iberian World, vol. 3, Viena \& Berlin, LIT Verlag, 2012, pp. 151-168.

Katzew, Ilona, New World Order. Casta Paintings and Colonial America, New York, Americas Society Art Gallery, 1996.

-, Casta Paintings. Images of race in eighteenth-century Mexico, New Haven and London, Yale University Press, 2004.

Konetzske, Richard, Colección de Documentos para la Historia de la Formación Social de Hispanoamérica, 1493-1810. vol. III, Tomo II, Madrid, Consejo Superior de Investigaciones Científicas. 
Kosselleck, R., «Historia de los conceptos y conceptos de historia», Ayer, vol. 53, n. ${ }^{\circ}$ 1, 2004, pp. 37-38.

Kuznesof, Elizabeth A., «Raza, clase y matrimonio en la Nueva España: estado actual del debate», en Gonzalvo Aizpuru, Pilar (coord.), Familias novohispanas. Siglos XVI al XIX, Seminario de Historia de la Familia, Centro de Estudios Históricos, México, El Colegio de México, 1991, pp. 373-386.

-, «Ethnic and Gender Influences on "Spanish" Creole Society», Colonial Latin American Review, vol. 4, n. ${ }^{\circ} 1,1995$, pp. 168-170.

Lavalle, Bernard, Recherches sur l'apparition de la conscience créole dans la vice-royauté du Pérou: l'antagonisme hispano-créole dans les ordres religieux (XVI-XVII siècle). Tesis Doctoral, Université de Bordeaux, III. Atelier National de Reproduction de Thèses, Université de Lille, 1982.

López Austin, Alfredo y Leonardo López Luján, El pasado indígena, México, Fondo de Cultura Económica [1996] 2001.

Marre, Diana, «Historia de la familia e historia social. La aplicación de la Pragmática Sanción de Carlos III en América Latina: una revisión», Quaderns de l'Institut Català d'Antropologia, núm. 10, pp. 217-249.

Martínez, M. ${ }^{a}$ Elena, Genealogical Fictions. Limpieza de sangre, Religion, and Gender in Colonial Mexico, Stanford, Stanford University Press, 2008.

Martínez Marín, Carlos, «La etnohistoria», en Perez Zevallos, Juan Manuel y José Antonio Pérez Gollán (comp.), La etnohistoria en Mesoamérica y los Andes, México, DF: Instituto Nacional de Antropología e Historia, 1987.

Martínez Mauri, Mónica. De Tule Nega a Kuna Yala. Mediación, territorio y ecología en Panamá, 1903-2004, Tesis Doctoral, École des Hautes Études en Sciences Sociales, Paris, 2007.

Menegus, Margarita, Los indios en la historia de México, México, Centro de investigación y docencia económicas \& Fondo de Cultura Económica, 2006.

Perez Zevallos, Juan Manuel, «La etnohistoria en México», Desacatos, n. ${ }^{\circ}$ 7, 2001, pp. 103-110.

Perez Zevallos, Juan Manuel y José Antonio Pérez Gollán (comp.), La etnohistoria en Mesoamérica y los Andes, México, DF: Instituto Nacional de Antropología e Historia, 1987.

Presta, Ana María, «Undressing the Coya and Dressing the Indian Woman: Market Economy, Clothing, and Identities in the Colonial Andes, La Plata (Charcas), Late Sixteenth and Early Seventeenth Centuries», Hispanic American Historical Review, vol. 90, n. ${ }^{\circ}$ 1, 2009, pp. 41-74.

Scott, James C., Weapons of the Weak. Everyday Forms of Peasant Resistance. New Haven, Yale University Press, 1985.

Stolcke, Verena, «Los mestizos no nacen, se hacen», en Verena Stolcke y Alexandre Coello (eds.), Identidades ambivalentes en América (siglos XVI-XXI), Barcelona, Bellaterra, 2008, pp. 17-58.

Thomson, Sinclair, «Was There Race in Colonial Latin America? Identifying Selves and Others in the Insurgent Andes», en Gotkowitz, Laura (ed.), Histories of Race and Racism. The Andes and Mesoamerica from Colonial Times to the Present, Durham \& London, Duke University Press, 2011, pp. 72-91.

Villella, Peter B., Indigenous Elites and Creole Identity in Colonial Mexico, 1500-1800, New York, Cambridge University Press, 2016.

Vinson, Ben III, Before Mestizaje: The Frontiers of Race and Caste in Colonial Mexico, New York, Cambridge University Press, 2017.

Viqueira Albán, Juan Pedro, Propriety and Permissiveness in Bourbon Mexico, Wilmington, Delaware, SR Book [1987] 1999. 
Zúñiga, Jean-Paul, «Cuerpos mestizos: genealogía y apariencia en la América hispánica». Ponencia presentada en la Jornada Internacional, El poder dels sistemes de classificació social. Sala de Juntes, Facultat de Filosofia i Lletres, Universitat Autònoma de Barcelona (UAB), 27 de octubre de 2011 . 